\title{
Gamificação no Processo de Ensino e Aprendizagem de Estudantes Surdos: uma revisão sistemática
}

Luiz Otavio Rodrigues Mendes, Universidade Estadual de Maringá, mendesluizotavio@ hotmail.com

Alcione José Alves Bueno, Universidade Tecnológica Federal do Paraná, alcioneab@gmail.com

Renata da Silva Dessbesel, Universidade Tecnológica Federal do Paraná, renatadessbesel@utfpr.edu.br

Sani de Carvalho Rutz da Silva, Universidade Tecnológica Federal do Paraná, sani@utfpr.edu.br

Resumo: Este estudo investiga as potencialidades e fragilidades da gamificação no processo de ensino e aprendizagem de estudantes surdos, a partir de uma revisão sistemática da literatura em artigos, teses e dissertações disponíveis nas bases de dados SciELO, Science Direct, ERIC, Scopus, Web Of Science, IEEE Xplore Digital Library, Advanced Technologies \& Aerospace Database, Teses e Dissertações CAPES e Biblioteca Digital Brasileira de Teses e Dissertações por meio dos descritores gamificação e surdos. Os resultados mostraram que as potencialidades em utilizar a gamificação na educação de alunos surdos estão relacionadas à utilização dos elementos dos games como agentes potencializadores (motivação, interação e aumento do interesse dos estudantes no desenvolvimento das tarefas) para contribuir em sua formação. A gamificação é abordada de forma inclusiva, auxiliando na compreensão de conceitos das diversas áreas do conhecimento e na aprendizagem da língua de sinais como meio de comunicação.

Palavras-chave: Tecnologia. Língua de Sinais. Motivação.

\section{Gamification in the Teaching-Learning Process of Deaf Students: a Literature Review}

\begin{abstract}
In a study, the potentialities and weakness of the gamification in the teachinglearning process of deaf students were researched. The systematic review of the literature was made using articles, theses and dissertations available in SciELO, Science Direct, ERIC, Scopus, Web Of Science, IEEE Xplore Digital Library, Advanced Technologies \& Aerospace Database, CAPES Theses and Dissertations and Digital Brazilian Library of Theses and Dissertation databases throughout the descriptors: gamification and deaf. The results showed that potentialities to use the gamification in deaf students' education are related to the use of elements of the games as booster agents to contribute in their education, such as motivation, interaction and the students interest increase in developing tasks. The gamification is approached in an inclusive way, helping to understand concepts to several areas of knowledge and learning the sign language as a mean of communication.
\end{abstract}

Keywords: Technology. Sign Language. Motivation.

\section{Introdução}

As diversas transformações que vêm ocorrendo na sociedade contemporânea têm como um dos principais fatores a exponencial utilização das tecnologias digitais, presentes nos mais diversos ambientes, inclusive no educacional. Apropriar-se de suas potencialidades nos processos de ensino e aprendizagem não é mais uma questão de 
quando utilizar, e sim, de como utilizar. Dentre as possibilidades, Groenwald (2016) destaca a importância do uso dos recursos tecnológicos na aprendizagem, de modo que as tecnologias oportunizam diferentes recursos didáticos para serem agregados à prática do professor, particularmente na educação especial.

A utilização desses recursos perpassa por diversos fatores que podem influenciar no processo de aprendizagem, como a disponibilização de recursos nas escolas, a acessibilidade e o acesso à internet. Kalinke (2014), ao discorrer sobre a falta de formação e materiais para o desenvolvimento das aulas, comenta que não é raro se encontrar professores que trabalham em mais de uma escola, transitando em distintas realidades. Diante da discrepância de recursos e seus impactos na vida dos alunos, consideramos a necessidade de uma busca por tecnologias educacionais acessíveis.

Dessa forma, as tecnologias podem ser aliadas dos professores, ao oferecer estratégias de ensino que sejam acessíveis a todos os públicos, possibilitando um ensino e aprendizagem com qualidade. Nesse sentido, Mendes (2019, p. 175) considera a gamificação como uma alternativa para sala de aula, por ser uma estratégia "[...] de ensino que transpõem (sic) os elementos dos games em ambientes educacionais, a partir da criatividade, promovendo atributos que contribuem para os processos de ensino e aprendizagem".

$\mathrm{Na}$ Educação de surdos as tecnologias oportunizam aos alunos superar os desafios da comunicação, como afirmam Nogueira, Carneiro e Silva (2018), permitindo acesso à informação, ampliando as relações sociais e dando-lhes mais autonomia. Além disso, os professores podem recorrer a esse recurso tornando as aulas visuais, trazendo benefícios no processo de aprendizagem de todos os estudantes, como afirmam Lacerda, Santos e Caetano (2013), a respeito da pedagogia visual. Os autores ainda destacam a necessidade de compreender que na educação de surdos a língua de sinais é o meio pelo qual os conceitos são organizados: "assim, para favorecer a aprendizagem do aluno surdo, não basta apenas apresentar os conteúdos em LIBRAS, é preciso explicar os conteúdos de sala de aula utilizando toda a potencialidade visual que essa língua tem" (LACERDA, SANTOS e CAETANO, 2013, p. 186).

Portanto, aproveitar as potencialidades da gamificação traz possibilidades para desenvolver os conceitos com os alunos surdos, oportunizando recursos visuais e interativos na sala de aula. Partindo do pressuposto que a gamificação pode ser uma forma de colaborar com o trabalho docente ao se apropriar dos elementos dos games, que são tecnologias presentes na realidade dos alunos de todos os níveis, este estudo pretende investigar as contribuições da gamificação no processo de ensino e aprendizagem de estudantes surdos.

\section{A gamificação como estratégia no processo de ensino e aprendizagem de alunos surdos}

Advindo do termo gamification, cunhado pelo britânico Nick Pelling em 2002, a gamificação, como é mais conhecida em trabalhos de língua portuguesa, tornou-se objeto de estudo de pesquisas acadêmicas somente nove anos após. No início, sua utilização esteve voltada para a área do marketing; a partir de 2013, no entanto, foram defendidas dissertações, teses e artigos nacionais sobre a temática no campo educacional, como um estudo realizado por Fardo em 2013.

De acordo com Fardo (2013), a utilização dos games esteve inicialmente ligada a uma estratégia que proporcionava engajar e motivar as pessoas a resolverem problemas. Atualmente, no entanto, entende-se que essa estratégia pode ser utilizada de diversas formas, apropriando-se dos elementos dos games para fazer as pessoas resolverem 
situações problemas (KIM et al., 2018). Quanto aos elementos dos games, Zichermann e Cunningham (2011, p. 36) consideram que os principais são: "[...] os pontos, tabelas de classificação, níveis, insígnias, desafios/missões, integração e loops de engajamento"; além desses, também para uma aplicação gamificada, é importante a utilização de uma narrativa (BUSARELLO, 2016a).

Referente a sua abordagem na educação, a gamificação tem uma flexibilização em seu uso, pois pode ser abordada de forma virtual, sendo empregada em sites, plataformas, aplicativos e de forma presencial, podendo ser utilizada em escolas com poucos recursos (MENDES, 2019). Assim, pode ser abordada nos diferentes contextos educacionais, como os destacados por Kalinke (2014) que podem contar ou não com recursos tecnológicos. É importante destacar também que não se especifica a aplicação gamificada para um conteúdo que será ensinado, mas para todo o processo. Nessa perspectiva, Esquivel (2017, p. 61) comenta que "o alvo da Gamificação é o processo de ensino e aprendizagem, e não determinado conteúdo", assim compreendemos que essa estratégia pode ser utilizada para o ensino de todos os alunos, em uma perspectiva inclusiva".

$\mathrm{O}$ decreto $\mathrm{n}^{\circ} 5.626$ de 22 de dezembro de 2005 destaca em seu art. 14 o acesso à comunicação, informação e educação, contemplando os conteúdos curriculares em todas as etapas de ensino. Desse modo, há uma legislação que garante a educação para as pessoas surdas; no entanto, Campos (2013) afirma que as leis ainda não foram contempladas em sua totalidade na prática.

A Lei de Inclusão da pessoa com deficiência (BRASIL, 2015) reafirma a Libras como meio de comunicação; percebe-se então que as condições de acesso à educação estão garantidas por leis, mas observa-se que a permanência e a oferta de uma educação efetiva e de qualidade ainda é emergente. Evidencia-se, por conseguinte, a necessidade da busca de estratégias que possam colaborar com a prática docente, para ser exercida sob os pressupostos da inclusão. Como destacado por Campos (2013), os surdos buscam no espaço escolar a valorização da cultura surda, a comunicação em língua de sinais, o respeito de sua identidade, a presença de intérpretes e as tecnologias especializadas de modo a contribuir com a educação.

Ao encontro dessa ideia, Dessbesel, Silva e Shimazaki (2018) enfatizam a importância da comunicação na educação de surdos por meio da língua de sinais nos espaços escolares inclusivos, de modo a efetivar o acesso à educação. Nesse sentido, a presença do Intérprete de Libras (ILS) muda a dinâmica da sala de aula. Lacerda, Santos e Caetano (2013) apontam a necessidade de o professor buscar práticas acessíveis, sendo fundamental a parceria entre o professor e o ILS: "Uma das formas de promover a parceria entre profissionais que beneficiam o aprendizado do aluno surdo, é envolver o ILS no planejamento das atividades" (LACERDA; SANTOS; CAETANO, 2013, p.196).

Assim sendo, o ambiente escolar precisa ser compreendido como um espaço de interação entre professor, aluno e ILS, como afirmam Soares e Sales (2018). Na educação de surdos a dinâmica curricular deve buscar alternativas metodológicas que considerem o contexto social, de forma que os estudantes se reconheçam como protagonistas do seu processo educativo. Nesse cenário a gamificação traz contribuições importantes para a sala de aula, como a interação entre os estudantes, o contexto lúdico e visual.

\section{Procedimentos metodológicos}

Este estudo trata-se de uma revisão sistemática da literatura, guiado segundo as orientações de Sampaio e Mancini (2007), que a definem como um método de pesquisa que sintetiza evidências de diversos estudos relacionados a um determinado tema, a partir de uma aplicação de métodos definidos e sistematizados de busca e análise crítica. 
A pesquisa segue cinco passos, a saber, definição da pergunta, busca pela evidência e identificação das bases de dados, revisão e seleção dos estudos, análise da qualidade metodológica dos estudos e apresentação dos resultados (SAMPAIO; MANCINI, 2007). Dessa forma, apresentam-se as etapas deste estudo de forma detalhada:

a) Definição da pergunta: "Quais as potencialidades e fragilidades da gamificação no processo de ensino e aprendizagem na educação de surdos";

b) Busca pela evidência: a pesquisa está situada na interface da gamificação e da educação de surdos; dessa forma foi definido o descritor gamification AND deaf * em língua inglesa por atender todas as bases usadas nesse estudo, sendo que a primeira palavra corresponde ao termo mais utilizado (MENDES, 2019) e o segundo atende as derivações da palavra. Com relação às bases de dados selecionadas, ficaram definidas as seguintes: SciELO, Science Direct, ERIC (Education Resources Information Center), Scopus, Web Of Science, IEEE Xplore Digital Library, Advanced Technologies \& Aerospace Database, e para teses e dissertações os dados foram buscados no Banco de Teses e Dissertações Capes e o Biblioteca Digital Brasileira de Teses e Dissertações - BDTD de modo a contemplar estudos nacionais e estrangeiros;

c) Revisão e seleção dos estudos: nesta etapa, após a seleção dos descritores nas respectivas bases, retornaram 79 pesquisas. Foi realizada a leitura do título e resumo de todos os estudos por dois pesquisadores e estabeleceram-se os seguintes critérios de exclusão: i) duplicação; ii) estudos teóricos, anais completos de eventos e entrevistas; iii) pesquisas relacionadas especificamente com games e serious games. Essa etapa resultou em 18 estudos. Para estes seguiram-se os critérios de inclusão i) pesquisas em espaços formais e não formais relacionadas à educação e ii) pesquisas que abordassem a educação de surdos. Ao final foram incluídas 14 pesquisas como amostra final para a análise, como detalhamos no quadro 1.

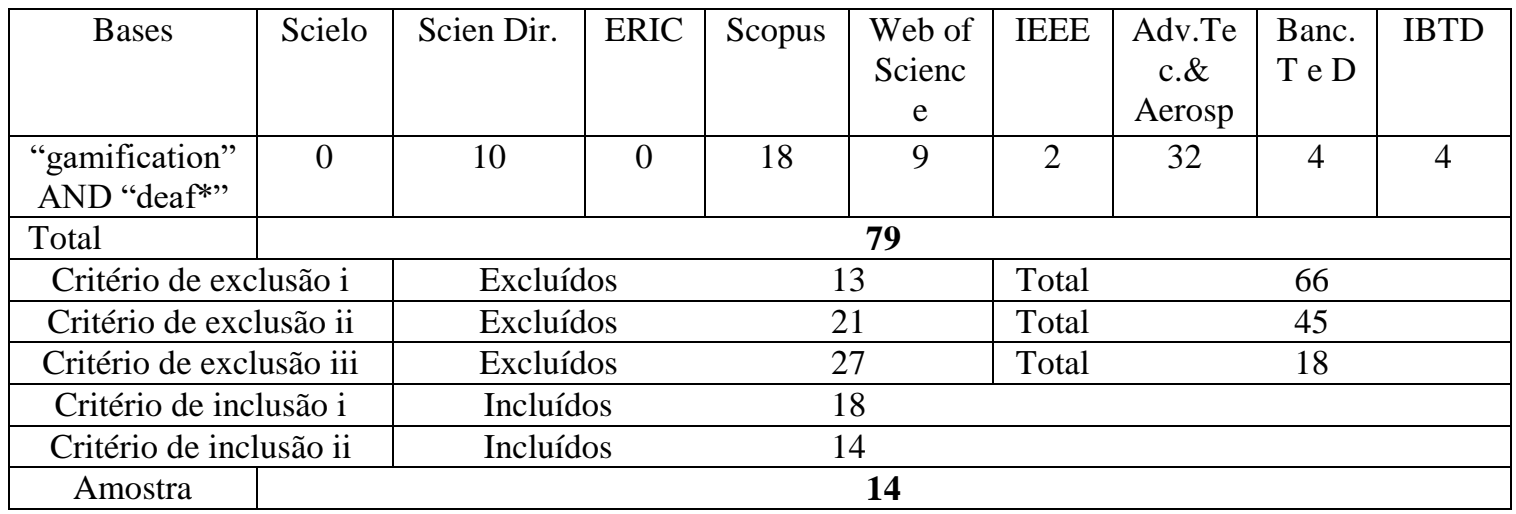

Quadro 1 - Revisão e seleção dos estudos.

Fonte: Elaborado pelos autores

d) Análise da qualidade metodológica dos estudos: nesta etapa foi realizada a análise crítica dos estudos com o objetivo de apontar possíveis contribuições quanto às potencialidades e fragilidades dos estudos e seus respectivos participantes. Para a extração dos dados dos artigos, foi organizado em uma planilha eletrônica a caracterização dos estudos quanto sua origem, ano de publicação e participantes da pesquisa, também as potencialidades e fragilidades identificadas pelos autores nos resultados dos estudos. Foi identificada mais de uma pesquisa dos autores Kamnardsiri et al. (2016), Rocha (2016) e Busarello (2016b) com relatos referentes ao mesmo experimento de ensino; assim, para a análise, considerou-se o texto com mais informações sobre o estudo e/ou do ano mais recente. 
e) Apresentação dos resultados: nesta etapa apresentaram-se os resultados da análise dos 10 artigos, momento quando foram caracterizadas as pesquisas e discutidas as potencialidades e fragilidades da gamificação na educação de surdos.

Na próxima seção apresentamos e discutimos os resultados da análise das pesquisas selecionadas como amostra final desta revisão sistemática da literatura.

\section{Resultados e discussões}

A Política Nacional de Educação Especial, na perspectiva da Educação Inclusiva (2008), considera a inclusão escolar de alunos com deficiência, transtornos globais do desenvolvimento e altas habilidades/superdotação no ensino regular e prevê uma série de medidas para que a inclusão seja efetivada, como a formação docente, o atendimento educacional especializado e as condições de acessibilidade. Nesse sentido, as tecnologias podem trazer para a sala de aula inclusiva diversas possibilidades, em específico no contexto da gamificação e educação de surdos.

Neste artigo, foram selecionadas 10 pesquisas, sendo uma tese, quatro dissertações e cinco artigos, provenientes de diferentes lugares, como representados na figura 1 . O tom da cor azul no mapa, escurece conforme a quantidade de produções aumenta. É possível perceber que $60 \%$ (6) se encontram no Brasil. Outrossim, ao compreender que as produções acadêmicas sobre a gamificação começaram a surgir em 2013, como o trabalho de Fardo (2013), é notável que essa relação entre gamificação e surdos seja um assunto mais recente ainda, conforme aponta o ano das produções que estão em uma faixa de 2016 a 2019.

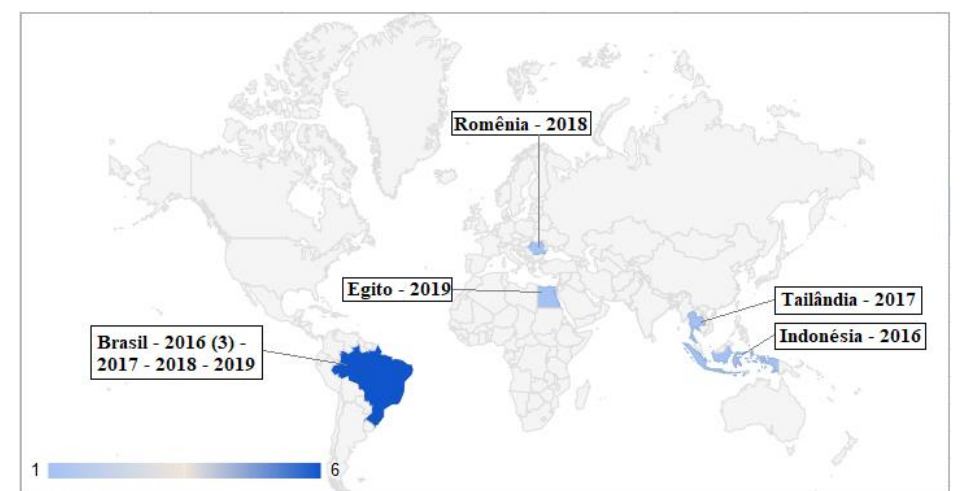

Figura 1 - Mapa global representando os países e anos em que foram selecionadas as produções. Fonte: Elaborado pelos autores

Como forma de caracterizar os estudos da amostra analisada, reuniu-se os estudos e os seus participantes. O perfil dos sujeitos das pesquisas é bastante diversificado, há pesquisas com alunos da Educação Básica como Anindhita e Lestari (2016), Busarello (2016b) e Rocha (2018), com estudantes da universidade como Ferreira et al. (2016) e Kamnardsiri et al. (2017), e pesquisas com professores que trabalham com alunos surdos, como o Bratu, Buică-Belciu e Caraman (2018). É possível perceber que a estratégia vem sendo trabalhada nas várias partes do mundo, bem como com os diversos níveis de ensino, o que permite inferir que esses são temas emergentes no campo educacional.

Outro ponto importante de destacar é como a gamificação vem sendo abordada. Mendes (2019) ressalta que há estratégias que se utilizam dos elementos dos games para serem empregadas em meios tecnológicos, considerando-as como a gamificação aplicada de forma virtual. Já as que não se utilizam de meios tecnológicos como smartphones, tablets, entre outros, são consideradas como a gamificação aplicada de forma presencial. 
A partir das análises das pesquisas, observou-se que apenas os trabalhos de Rios (2016) e Bratu, Buică-Belciu e Caraman (2018) não definem especificamente se a estratégia foi aplicada somente de forma virtual ou também presencial, por ser uma proposta de atividade; para os outros foi empregada de forma virtual.

Com relação às potencialidades e fragilidades da utilização da gamificação na educação de estudantes surdos, apresentam-se, no quadro 2, os resultados com base em leituras sobre os textos.

\begin{tabular}{|c|c|c|}
\hline Estudos & Potencialidade & Fragilidade \\
\hline $\begin{array}{l}\text { Anindhita e Lestari } \\
\text { (2016) }\end{array}$ & $\begin{array}{l}\text { Os elementos gamificados incentivaram os alunos a utilizar a } \\
\text { interface para estudarem inglês. }\end{array}$ & Não foi apontado. \\
\hline Ferreira et al. (2016) & $\begin{array}{l}\text { Destacaram que os elementos gamificados possibilitaram } \\
\text { interação, envolvimento e imersão do usuário ao conteúdo. }\end{array}$ & Não foi apontado. \\
\hline Busarello (2016b) & $\begin{array}{l}\text { A estratégia deixou mais dinâmica a atividade. Os elementos } \\
\text { gamificados metas e regras foram eficientes para a interação } \\
\text { com os surdos. }\end{array}$ & Não foi apontado. \\
\hline Rios (2016) & $\begin{array}{l}\text { A não seriedade e a voluntariedades que são elementos } \\
\text { advindos dos games são importantes para o sucesso de } \\
\text { aplicações gamificadas. }\end{array}$ & Não foi apontado. \\
\hline $\begin{array}{l}\text { Kamnardsiri et al. } \\
\text { (2017) }\end{array}$ & $\begin{array}{l}\text { O desempenho de alunos que utilizaram os elementos dos } \\
\text { games foi maior do que alunos que não utilizaram. }\end{array}$ & Não foi apontado. \\
\hline Nascimento (2017) & $\begin{array}{l}\text { Os elementos gamificados utilizados foram } 100 \% \text { indicados } \\
\text { por professores de Libras para serem utilizados nas aulas. } \\
\text { Proporcionou motivação, engajamento e interação dos } \\
\text { usuários. }\end{array}$ & $\begin{array}{l}\text { Destacam que foi difícil } \\
\text { definir como as premiações } \\
\text { seriam entregues para não } \\
\text { perder a atratividade do } \\
\text { trabalho. }\end{array}$ \\
\hline $\begin{array}{l}\text { Bratu, Buică-Belciu e } \\
\quad \text { Caraman (2018) }\end{array}$ & $\begin{array}{l}\text { Acessibilidade ao conteúdo. Organização e racionalização do } \\
\text { uso do tempo de aprendizado. Os alunos ficaram mais ativos } \\
\text { no desenvolvimento das atividades escolares. }\end{array}$ & $\begin{array}{l}\text { Concorrência } \\
\text { alunos. }\end{array}$ \\
\hline Rocha (2018) & $\begin{array}{l}\text { O elemento de ranking aumentou o interesse em } 70 \% \text { dos } \\
\text { estudantes. Aumento de interesse e motivação. }\end{array}$ & Não foi apontado. \\
\hline Shohieb (2019) & $\begin{array}{l}\text { Possibilidade de se trabalhar em disciplinas difíceis como a } \\
\text { Matemática. }\end{array}$ & Não foi apontado. \\
\hline Rodrigues (2019) & $\begin{array}{l}\text { Os elementos dos games proporcionaram a competitividade } \mathrm{e} \\
\text { a auto superação. }\end{array}$ & Não foi apontado. \\
\hline
\end{tabular}

Quadro 2 - Potencialidades e fragilidades da gamificação na educação de surdos

Fonte: Elaborado pelos autores

Com base na observação do quadro 2, é possível perceber que dentre os trabalhos, a maioria não apresentou possíveis fragilidades em relação específica à utilização da gamificação. Mas dentre as duas pesquisas que retrataram fragilidades, percebe-se que para Nascimento (2017) elas estão relacionadas a uma dificuldade procedimental, no que concerne à utilização dos elementos dos games na aplicação. Ratificando a ideia, Mendes (2019, p.41) apontou sugestões de como aplicar a gamificação, destacando aspectos a serem definidos previamente: "[...] o escopo, o conhecimento que precisará ser ensinado, os comportamentos dos alunos e quais elementos (mecânicas, narrativa, etc.) serão utilizados". Os elementos, por exemplo, as "premiações" citadas na pesquisa Nascimento (2017), devem ser planejadas na aplicação da gamificação.

As fragilidades apontadas por Bratu, Buică-Belciu e Caraman (2018) merecem atenção, pois a competição quando trabalhada sem a mediação dos professores pode fazer que a atividade não seja profícua. De acordo com Kapp (2012), a competição deve ser abordada como superação. À vista disso, compreende-se que a mediação do professor é ponto fundamental para haver uma competição colaborativa. Assim, dependendo de como ela é trabalhada, pode ser considerada como um ponto positivo, como destacado por Rodrigues (2019), em sua pesquisa. 
A respeito das potencialidades, é possível perceber que todos os trabalhos destacaram ao menos uma, o que nos permite inferir que a gamificação no contexto educacional atua como potencializador no processo de ensino e aprendizagem dos estudantes. A gamificação, embasada nos pressupostos descritos por Kapp (2012) como uma estratégia que possibilita a motivação e o engajamento dos usuários, é destacada conforme os trabalhos de Nascimento (2017), Bratu, Buică-Belciu e Caraman (2018), e Rocha (2018), confirmando a potencialidade da estratégia. No entanto, a estratégia não se resume a apenas isso; Kim et al. (2018) abordam uma concepção mais geral, não se limitando à motivação e ao engajamento.

Dessa forma, evidencia-se que esse novo olhar sobre a gamificação se faz eficaz, visto que em Ferreira et al. (2016) são explorados os conceitos de gamificação em uma plataforma, com objetivo de estabelecer envolvimento e imersão dos estudantes no desenvolvimento dos conteúdos. A interação entre os alunos, que também é destacada em Busarello (2016b) e Nascimento (2017), vem reforçar esse entendimento defendido por Kim et al. (2018).

Além disso, percebe-se que a utilização da gamificação possibilitou um papel inclusivo, levando em conta as diferenças individuais como é garantindo nas leis e decretos com relação à inclusão. Esse entendimento é perceptível na pesquisa de Bratu, Buică-Belciu e Caraman (2018), a qual elenca que um dos benefícios da utilização da gamificação diz respeito à acessibilidade do conteúdo, considerando ainda que os alunos ficaram mais ativos na atividade gamificada. Concorda-se com Lacerda, Santos e Caetano (2013) que afirmam que o papel dos professores na educação de surdos é considerar as singularidades, dar sentido à aprendizagem na construção dos conceitos, promovendo a equidade entre os estudantes com relação ao acesso ao conhecimento.

Motivação e entusiasmo também são apontados na utilização da estratégia por Rocha (2018), ao considerar que houve um aumento do interesse pelos participantes, em específico por utilizarem elementos tecnológicos. Em Rodrigues (2019), o autor aponta como resultado a autosuperação dos alunos surdos ao passarem por atividades gamificadas, possibilitando assim que essa estratégia possa ser trabalhada de forma inclusiva.

De acordo com Mendes (2019), a gamificação é compreendida como uma estratégia para ser utilizada nos ambientes formais e não formais de ensino, de modo que ela pode ter suas influências nos processos de ensino e aprendizagem de forma inclusiva. Percebese esse entendimento nas pesquisas analisadas, pois a estratégia vem sendo utilizada para o ensino de conteúdos entendidos como difíceis a todos os alunos, conforme apontou a Shohieb (2019) ao enfatizar a matemática e em Ferreira et al. (2016) ao desenvolver a gamificação para o ensino do conteúdo de geometria descritiva, possibilitando assim alcançar os alunos em suas mais diversas especificidades. Ao encontro disso, Dessbesel, Silva e Shimazaki (2018) observaram que as pesquisas com relação ao ensino de matemática na educação de surdos apresentaram diversos recursos como mediadores do processo de aprendizagem, incluindo o uso de recursos tecnológicos como os softwares.

Da mesma forma, a pesquisa de Busarello (2016b), ao se apropriar das mecânicas dos games, o que se considera como um elemento presente na gamificação, aponta que "estas mecânicas formatam o elemento prático do sistema, que exigem maior envolvimento do aluno nas regras do sistema e englobam os conceitos de geometria descritiva" (p. 272), vindo assim a contribuir de forma a transformar tarefas cansativas em algo motivador. Outrossim, também foi utilizada para o ensino do inglês como aponta Anindhita e Lestari (2016), reforçando o entendimento de Esquivel (2017) em que a gamificação pode ser utilizada nos mais diversos processos de ensino e aprendizagem, e não apenas, para determinado conteúdo. 
Além dessas disciplinas de matemática e inglês, observa-se que a gamificação vem sendo utilizada como um importante elemento na criação de sistemas de comunicação entre surdos e entre surdos e ouvintes por meio da língua de sinais, como mostram os estudos de Kamnardsiri et al. (2017), Rocha (2018), e Shohieb (2019), que têm esse propósito em relação à língua de sinais, superando as barreiras de comunicação, como explicado por Campos (2013, p. 48): "a língua de sinais permite ao ser surdo expressar seus sentimentos e visões sobre o mundo, sobre significados, de forma mais completa e acessível".

De acordo com Mendes (2019), a gamificação traz possibilidades de ser abordada nos diferentes contextos e contribuir no processo de construção dos conceitos, como indicado por Bratu, Buică-Belciu e Caraman (2018) ao retratarem que a utilização da estratégia possibilitou uma melhor organização e racionalização do uso do tempo, fatores relevantes para o trabalho do professor. Nesse sentido, é possível reforçar o papel da gamificação como uma estratégia inclusiva, que leva em consideração as especificidades dos alunos.

\section{Considerações Finais}

Com o advento das tecnologias, a presença de elementos tecnológicos nos ambientes formais e não formais de ensino vem se tornando a cada dia mais comum; são diversas as possibilidades que podem surgir ao se apropriar dessas ferramentas tais como, a plotagem de gráficos, envio de feedbacks imediatos, entre outras. Ao compreender a gamificação como uma estratégia que pode ser utilizada em ambiente com maior ou menor presença das tecnologias, e de forma inclusiva, como é retratado por Mendes (2019), esta pesquisa buscou investigar as potencialidades e fragilidades da gamificação no processo de ensino e aprendizagem de estudantes surdos.

Os dados analisados sobre a utilização da gamificação revelam que esta pode ser utilizada em sua totalidade de forma virtual, ainda mais que os mais diversos públicos desde alunos da Educação Básica, Ensino Superior e até mesmo professores podem fazer uso de suas potencialidades. As fragilidades ao utilizar a gamificação na formação de alunos surdos estão relacionadas ao planejamento de estratégias, que podem ser superadas ao estabelecer previamente as condições e ferramentas que serão exploradas.

Outra fragilidade está relacionada à competição entre os alunos, no entanto, compreendemos que ela deve ser abordada conforme defende Kapp (2012), de forma mediada. As potencialidades em utilizar a gamificação na formação de alunos surdos estão relacionadas à utilização dos elementos dos games como agentes potencializadores para contribuir na formação dos alunos, tais como motivação, interação e aumento do interesse dos estudantes no desenvolvimento das tarefas. Reforça-se a gamificação como uma estratégia que pode ser trabalhada de forma inclusiva, auxiliando na compreensão de conceitos das diversas áreas do conhecimento e na aprendizagem da língua de sinais como meio de comunicação.

Compreende-se, portanto, a gamificação como uma importante ferramenta para ser utilizada na formação de alunos surdos, conforme se torna possível perceber suas potencialidades. Mesmo havendo algumas fragilidades, percebe-se que elas podem ser contornadas. Por fim, entende-se que há um espaço aberto para a pesquisa na interface gamificação e alunos surdos, bem como concebe-se essa estratégia como inclusiva e que pode ser trabalhada na diversidade.

\section{Referências Bibliográficas}


ANINDHITA, V.; LESTARI, D. P. Designing interaction for deaf youths by using usercentered design approach. In: International Conference on Advanced Informatics: Concepts, Theory and Application, Anais... IEEE, 2016. p. 1-6.

BRASIL. Decreto n ${ }^{\circ} 5626$ de 22 de dezembro de 2005. Regulamenta a Lei no 10436 de 24 de abril de 2002, que dispõe sobre a Língua Brasileira de Sinais e o art. 18 da Lei no 10098, de 19 de dez. 2000. Diário Oficial da União, Brasília, DF, 23 de dez. 2005.

BRASIL. Ministério da Educação. Secretaria de Educação Especial. Política nacional de educação especial na perspectiva da educação inclusiva. Documento nomeado pela Portaria $n^{\circ} 555 / 2007$, prorrogada pela Portaria $n^{\circ}$ 948/2007, entregue ao Ministro da Educação em 07 de janeiro de 2008. Brasília, DF, 2008.

BRASIL. Lei n ${ }^{\circ}$ 13.146, de 6 de julho de 2015. Institui a Lei Brasileira de Inclusão da Pessoa com Deficiência (Estatuto da Pessoa com Deficiência). Diário Oficial da União: Brasília, DF, 07 jul. 2015.

BRATU, M.; BUICĂ-BELCIU, C.; CARAMAN, D. Teachers' Perspective on Using of New Pedagogical Approaches for Students with Hearing Impairment. E-Learning \& Software for Education, v. 1, 2018.

BUSARELLO, R. I. Gamification: princípios e estratégias. São Paulo: Pimenta Cultural, 2016a.

BUSARELLO, R. I. Gamificação em histórias em quadrinhos hipermídia: diretrizes para construção de objeto de aprendizagem acessível. 2016. 352 p. Tese (Doutorado em Engenharia e Gestão do Conhecimento) - Universidade Estadual de Santa Catarina, Florianópolis, SC, 2016b.

CAMPOS, M. de L. I. L. Educação inclusiva para surdos e as políticas vigentes. In: LACERDA, C. B. F. de; SANTOS, L. F. dos. Tenho um aluno surdo e agora? Introdução à LIBRAS e Educação de surdos. São Carlos: EdUFSCar, 2013, p.37-62.

DESSBESEL, R. da S.; SILVA, S. de C. R. da; SHIMAZAKI, E. M. O processo de ensino e aprendizagem de Matemática para alunos surdos: uma revisão sistemática. Ciência \& Educação (Bauru), v. 24, n. 2, p. 481-500, 2018.

ESQUIVEL, H. C. R. Gamificação no ensino da matemática: uma experiência no ensino fundamental. 2017. 64F. Dissertação (Mestrado em Rede Nacional) Universidade Federal Rural do Rio de Janeiro, 2017.

FARDO, M. L. A Gamificação como estratégia pedagógica: Estudo de elementos dos games aplicados em processos de ensino. 2013. 106 f. Dissertação (Mestrado em Educação). Universidade de Caxias do Sul, Caxias do Sul -RS, 2013.

FERREIRA, P. G.; ANDRADE, R.; OLIVEIRA, S.; ULBRICHT, V.R. Learning Object Design for Teaching Descriptive Geometry: A Study from the Perspective of Gamification and Accessibility. In: International Conference on Universal Access in Human-Computer Interaction. Springer, Cham, 2016. p. 38-48.

GROENWALD, C. L. O. Inclusão e Educação Matemática. In: MENDES, E. G.; ALMEIDA, M. A. (org.) Inclusão escolar e educação especial no Brasil: entre o instituído e o instituinte. Marília: ABPEE, 2016. p. 135-148.

KALINKE, M. A. Tecnologias no ensino: a linguagem matemática na web. Curitiba: CRV, 2014. 
KAMNARDSIRI, T.; HONGSIT, L., KHUWUTHYAKORN, P; WONGTA, N. The Effectiveness of the Game-Based Learning System for the Improvement of American Sign Language Using Kinect. Electronic Journal of e-Learning, v. 15, n. 4, p. 283296, 2017.

KAPP, K. M. The gamification of learning and instruction. San Francisco: Wiley, 2012.

KIM, S.; Song, K.; Lockee, B.; Burton, J. Gamification in Learning and Education: Enjoy Learning Like Gaming. Switzerland: Springer, 2018. 164 p.

LACERDA, C. B. F. de.; SANTOS, L. F. dos., CAETANO, J. F. Estratégias metodológicas para o ensino de alunos surdos. In: LACERDA, C. B. F. de; SANTOS, L. F. dos. Tenho um aluno surdo e agora? Introdução à LIBRAS e Educação de surdos. São Carlos: EdUFSCar, 2013, p.185-200.

MENDES, L. O. R. A gamificação como estratégia de ensino: a percepção dos professores de matemática. 2019. 213 f. Dissertação (Mestrado em Ensino de Ciências e Educação Matemática) - Universidade Estadual de Ponta Grossa, Ponta Grossa, 2019.

NASCIMENTO, D. S. do. Um método para desenvolvimento de ambientes de aprendizagem da Língua Brasileira de Sinais (LIBRAS) baseado em Gamification. 2017. Dissertação (Mestrado). Universidade Federal de Pernambuco, 2017.

NOGUEIRA, C. M. I.; CARNEIRO, M. I. N.; SILVA, T. S. A. da. O uso social das tecnologias de comunicação pelo surdo: limites e possibilidades para o desenvolvimento da linguagem. Revista Pesquisa Qualitativa. São Paulo, v.6, n.12, p.470-497, 2018.

RIOS, L. T. R. A gamificação no processo de aprendizagem de LIBRAS. 2016. Dissertação (Mestrado em Tecnologia da Inteligência e Design Digital) - Pontifícia Universidade Católica, São Paulo, 2016.

ROCHA, P. S. R. Uma ferramenta computacional gamificada como estratégia de apoio para aprendizagem da língua brasileira de sinais (Libras). Dissertação (Mestrado em Ciência da Computação). Universidade Federal Rural do Semiárido, Mossoró-RN, 2018.

RODRIGUES, T. T. O jogo digital como recurso didático na alfabetização cartográfica de alunos surdos e deficientes auditivos em Santa Maria, RS/Brasil. (2019). Dissertação (Mestrado em Geografia). Universidade Federal de Santa Maria, RS, 2019.

SAMPAIO, R.F.; MANCINI, M. C. Estudos de revisão sistemática: um guia para síntese criteriosa da evidência científica. Revista Brasileira de Fisioterapia, v. 11, n.1, p. 83-89, São Carlos, 2007.

SHOHIEB, S. M. A Gamified e-Learning Framework for Teaching Mathematics to Arab Deaf Students: Supporting an Acting Arabic Sign Language Avatar. Ubiquitous Learning: An International Journal, v.12, n.1, p. 55-70, 2019.

SOARES, M. E.; SALES, E. R. Das memórias às ideias: Orientações sobre a visualidade na Educação Matemática para surdos. REPPE - Revista de Produtos Educacionais e Pesquisas em Ensino, Cornélio Procópio, PR, v. 2, n. 1, 2018.

ZICHERMANN, G; CUNNINGHAM, C. Gamification by Design: Implementing Game Mechanics in Web and Mobile Apps. Sebastopol: O'reilly, 2011. 